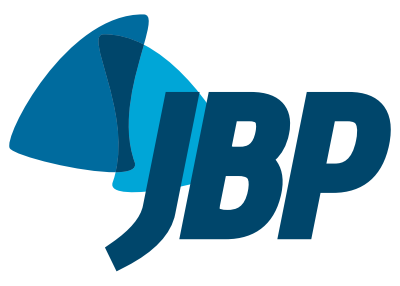

\title{
Translation and cultural adaptation of a specific instrument for measuring asthma control and asthma status: the Asthma Control and Communication Instrument
}

\author{
Michelle Gonçalves de Souza Tavares ${ }^{1,2}$, Carolina Finardi Brümmer \\ Gabriela Valente Nicolau ${ }^{3}$, José Tavares de Melo Jr ${ }^{1}$, Nazaré Otilia Nazário1,3, \\ Leila John Marques Steidle ${ }^{3}$, Cecília Maria Patino ${ }^{4}$, \\ Marcia Margaret Menezes Pizzichini1,3, Emílio Pizzichini ${ }^{1,2}$
}

1. Programa de Pós-Graduação em Ciências Médicas, Universidade Federal de Santa Catarina - UFSC Florianópolis (SC) Brasil.

2. Universidade do Sul de Santa Catarina UNISUL - Tubarão (SC) Brasil.

3. Núcleo de Pesquisa em Asma e Inflamação das Vias Aéreas - NUPAIVA - Universidade Federal de Santa Catarina, Florianópolis (SC) Brasil.

4. Department of Preventive Medicine, Keck School of Medicine, University of Southern California, Los Angeles (CA) USA.

Submitted: 31 October 2016

Accepted: 6 March 2017

Study carried out at the Universidade Federal de Santa Catarina - UFSC -

Florianópolis (SC) Brasil.

\begin{abstract}
Objective: To translate the Asthma Control and Communication Instrument (ACCI) to Portuguese and adapt it for use in Brazil. Methods: The ACCI was translated to Portuguese and adapted for use in Brazil in accordance with internationally accepted guidelines. The protocol included the following steps: permission and rights of use granted by the original author; translation of the ACCI from English to Portuguese; reconciliation; backtranslation; review and harmonization of the back-translation; approval from the original author; review of the Portuguese version of the $\mathrm{ACCl}$ by an expert panel; cognitive debriefing (the clarity, understandability, and acceptability of the translated version being tested in a sample of the target population); and reconciliation and preparation of the final version. Results: During the cognitive debriefing process, 41 asthma patients meeting the inclusion criteria completed the $\mathrm{ACCl}$ and evaluated the clarity of the questions/ statements. The clarity index for all $\mathrm{ACCl}$ items was $>0.9$, meaning that all items were considered to be clear. Conclusions: The $\mathrm{ACCl}$ was successfully translated to Portuguese and culturally adapted for use in Brazil, the translated version maintaining the psychometric properties of the original version. The $\mathrm{ACCl}$ can be used in clinical practice because it is easy to understand and easily applied.
\end{abstract}

Keywords: Asthma/classification; Asthma/prevention \& control; Surveys and questionnaires.

\section{INTRODUCTION}

In its latest report, the Global Initiative for Asthma(1) defined asthma as "a heterogeneous disease, usually characterized by chronic airway inflammation. It is defined by the history of respiratory symptoms such as wheeze, shortness of breath, chest tightness and cough that vary over time and in intensity, together with variable expiratory airflow limitation".

The 2013 Brazilian National Ministry of Health and Brazilian Institute of Geography and Statistics National Health Survey showed that asthma affects 6.4 million Brazilians over 18 years of age.(2) The prevalence of asthma was found to be $39 \%$ higher in females than in males, approximately 3.9 million females and 2.4 million males having reported a diagnosis of asthma. The National Health Survey was the first study to assess the occurrence of asthma in adults in Brazil. The World Health Organization estimates that $\mathbf{3 0 0}$ million children and adults worldwide have asthma. ${ }^{(3)}$ Asthma accounts for a significant number of hospitalizations in Brazil. Between January and November of 2014, there were 105,500 hospitalizations for asthma, which cost 57.2 million Brazilian reals to the public health care system, according to data from the Brazilian Unified Health Care System Hospital Information Service. ${ }^{(4)}$

The goal of asthma treatment is to control the disease, ${ }^{(1,5,6)}$ asthma control being defined as the extent to which the manifestations of asthma are reduced or removed by treatment. Asthma control can be divided into two domains: current impairment and future risk. ${ }^{(1)}$ The domain of current impairment includes symptoms, use of rescue medication, physical activity, and lung function. The domain of future risk includes accelerated loss of lung function over time, exacerbations, and treatment side effects. ${ }^{(1,7)}$

In recent years, several studies ${ }^{(8-13)}$ have highlighted the importance of standardizing the assessment of asthma control. As a result, several instruments have been validated and culturally adapted for use in Brazil, including the Asthma Control Test, ${ }^{(12)}$ the Asthma Control Questionnaire, ${ }^{(11)}$ and the Asthma Control Scoring System. ${ }^{(13,14)}$ However, none of the aforementioned instruments was developed specifically for or validated for use in racially diverse populations of asthma patients,

Correspondence to:

Emilio Pizzichini. NUPAIVA, Hospital Universitário, Campus Universitário, Rua Professora Maria Flora Pausewang, s/n, Trindade, CEP 88036-800, Florianópolis, SC, Brasil. Tel./Fax: 5548 3234-7711. E-mail: emiliopizzichini@gmail.com

Financial support: Carolina Finardi Brümmer and Gabriela Valente Nicolau received financial support from the Brazilian Conselho Nacional de Desenvolvimento Científico e Tecnológico (CNPq, National Council for Scientific and Technological Development) Programa Institucional de Bolsas de Iniciação Cientifica (PIBIC, Institutional Program for Young Investigator Grants). 
as is the case in Brazil. ${ }^{(8)}$ This is important in countries such as Brazil because certain colloquial terms might differ among regions within the same country, ${ }^{(15)}$ as well as among immigrants from different countries.(16) Therefore, a questionnaire should be used only in the population for which it has been developed and validated. (17) This bias is of vital importance if the objective of a given epidemiological study is to compare different locations and cultures. ${ }^{(15)}$

With the objective of assessing asthma control and communication, Patino et al. ${ }^{\left({ }^{8}\right)}$ developed the Asthma Control and Communication Instrument (ACCI). The ACCI is a 12-item self-report questionnaire for asthma patients over 12 years of age. It takes 5-7 minutes to complete. The ACCI has four domains of assessment of asthma activity: acute care, also known as "risk"; bother; control; and direction of symptoms. In addition, it has one domain for assessment of patient adherence to medication and one domain designed to improve physician-patient communication (one open-ended question). The response choices to questions are sequentially color coded from green (best) to yellow, orange, and red (worst). The ACCI control domain is the only multi-item component of the questionnaire that is scored by the physician, according to patient responses.

The ACCI provides three alternative scoring formats that can be used on the basis of physician preference. The first method, designated categories, classifies patients into four categories ranging from mildintermittent to severe-persistent, the former indicating better asthma status and the latter indicating poorer asthma status. Consistent with asthma guidelines, ${ }^{(1)}$ the control category is assigned by the most severe response among the five ACCI control items. Patients with intermittent symptoms are considered to be "controlled", whereas those with persistent symptoms are considered to be "uncontrolled". The second method, designated sum score, uses a summation of the five ACCI control items individually coded from 0 to 4 (the exception being the attack item, which is coded from 0 to 3 ). The sum score ranges from 0 (best) to 19 (worst). The third method, designated problem index, dichotomously rates each item as a control problem (yes or no), the values of which are then summed to provide a problem index ranging from 0 (no control problems) to 5 (five control problems).

Because of its characteristics, the ACCI is a promising clinical tool for measuring asthma control during routine health care and for research; however, in order to be used in Brazil, the ACCI had to be translated to Portuguese and culturally adapted for use in the country. Translation and cultural adaptation are needed in order to preserve the essential features of the original instrument (which was developed for ethnically diverse populations and therefore addresses social and cultural diversity) and prevent misunderstandings arising from literal translations. Therefore, the objective of the present study was to translate the $\mathrm{ACCI}^{(8)}$ to Portuguese and adapt it for use in Brazil.

\section{METHODS}

This was a methodological study aimed at translating the ACCI to Portuguese and adapting it for use in Brazil, the ACCI being an instrument specifically designed to measure asthma control and communication. ${ }^{(8)}$ The study was approved by the Human Research Ethics Committee of the Federal University of Santa Catarina, located in the city of Florianópolis, Brazil, and was conducted in accordance with established ethical principles. Consent was obtained from the first author of the original instrument, who participated in the validation process.

The ACCI was translated to Portuguese and adapted for use in Brazil in accordance with the guidelines established by Mapi and those used in other studies. $(15,16,18,19)$ Figure 1 illustrates the steps involved in the process.

The process of translation and cultural adaptation of a questionnaire involves determining the acceptability, understandability, and clarity of the translated version in a sample of the target population; this step of the process is designated cognitive debriefing. ${ }^{(16-19)}$ A total of 41 asthma patients participated in the cognitive debriefing process in the present study. Participants were consecutively recruited from among patients routinely followed at the Pulmonology Outpatient Clinic of the Federal University of Santa Catarina University Hospital or at a private respiratory medicine clinic, both of which are located in the city of Florianópolis, Brazil. All of the patients who met the inclusion criteria and gave written informed consent participated in the study. The inclusion criteria were as follows: being in the 18- to 70-year age bracket; having asthma (i.e., having had episodes of wheezing, chest tightness, and dyspnea in the previous year); having a diagnosis of asthma objectively confirmed by reversible airflow limitation ( $a>15 \%$ increase in $\mathrm{FEV}_{1}$ after inhalation of a short-acting bronchodilator, with an $\mathrm{FEV}_{1}$ of $<70 \%$ of predicted or an $\mathrm{FEV}_{1} / \mathrm{FVC}$ ratio of $<70 \%$ ) or airway hyperresponsiveness, as detected by methacholine challenge testing - the provocative concentration of methacholine causing a $20 \%$ drop in $\mathrm{FEV}_{1}$ being $<8$ $\mathrm{mg} / \mathrm{mL}$-with an $\mathrm{FEV}_{1}>70 \%$ of predicted; currently receiving pharmacological treatment; having been clinically stable for at least one month, independently of the presence of atopy; and being a nonsmoker or having been a former smoker for more than 1 year, with a smoking history of $<10$ pack-years. The exclusion criteria were as follows: having other known lung diseases (including chronic bronchitis, COPD, and pneumonia); having severe disease affecting systems other than the respiratory system; and having any psychiatric or cognitive disorder that could confound the results.

Given that the present study does not permit a statistical analysis, the data are reported as absolute numbers and proportions, as means and standard deviations, or as medians and interquartile ranges. Statistical analysis was used in order to determine the demographic and clinical characteristics of the participants. 


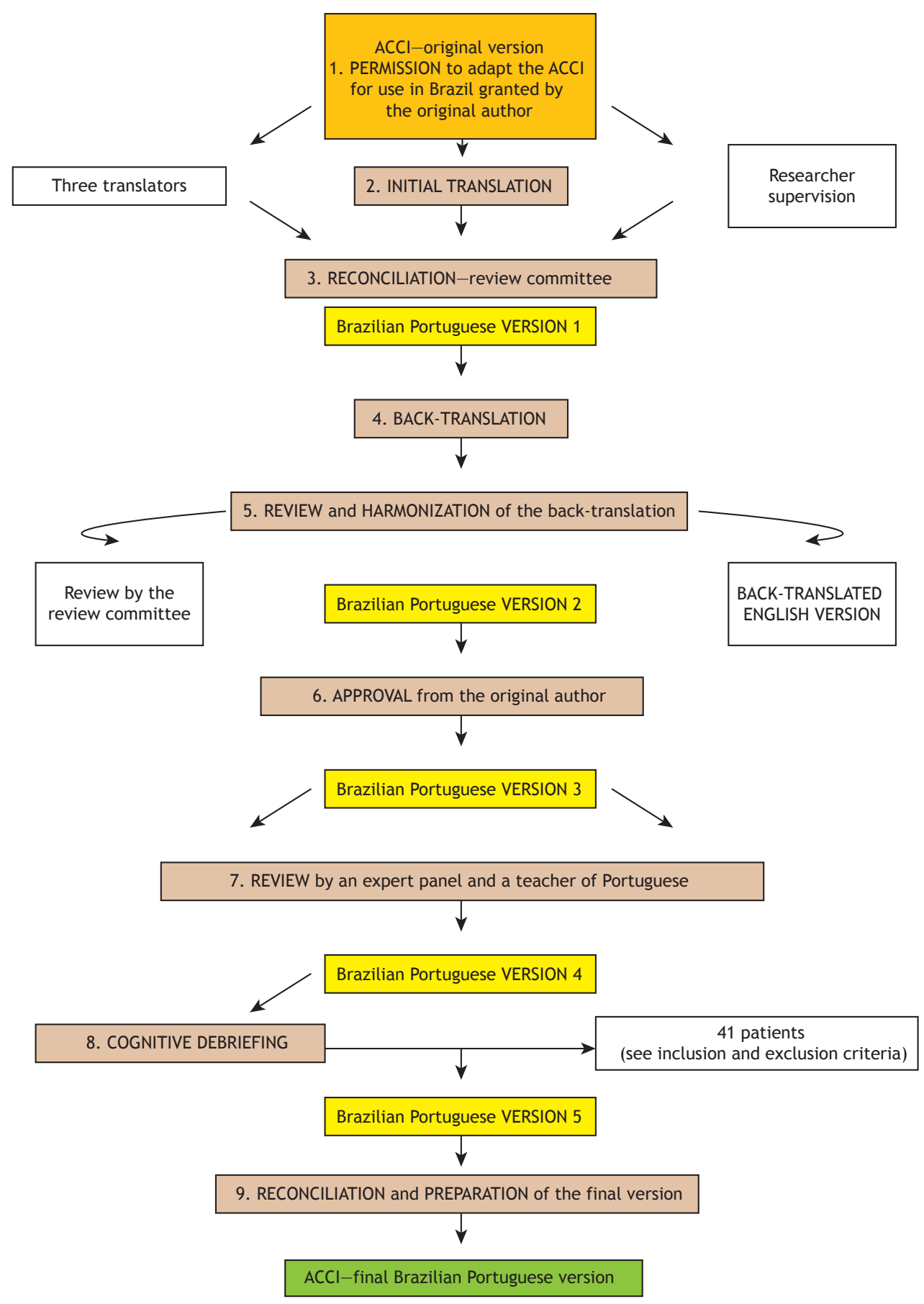

Figure 1. Summary of the process of cross-cultural adaptation of the Asthma Control and Communication Instrument (ACCI) for use in Brazil.

Cultural adaptation of the ACCI was done in accordance with internationally accepted guidelines, ${ }^{(16-19)}$ and the protocol included the following steps (Figure 1):

- preparation-permission to translate the ACCI to Portuguese and adapt it for use in Brazil obtained from the first author of the original version

- translation of the ACCI from English to Portuguese by three translators fluent in English (all three translations having been performed independently)

- reconciliation-analysis and comparison of the three versions by a review committee comprising experts in the area, resulting in the first Brazilian Portuguese version of the ACCI (version 1)

- back-translation-literal translation of version 1 to English by a teacher of English (a native speaker of English fluent in Portuguese) 
- review and harmonization of the back-translationreview of the back-translation to English, resulting in the second Brazilian Portuguese version of the ACCI (version 2)

- analysis of version 2 by the original author, the third Brazilian Portuguese version of the ACCI (version 3) being arrived at after the corrections and suggestions made by the original author

- review of version 3 by three bilingual pulmonologists, the fourth Brazilian Portuguese version of the ACCI (version 4) being arrived at

- cognitive debriefing-the clarity, understandability, and acceptability of version 4 being tested in a sample of the target population (41 asthma patients selected during routine outpatient visits, meeting the inclusion criteria, and giving written informed consent)

- analysis of the comments made by participants, resulting in the fifth Brazilian Portuguese version of the ACCI (version 5), which included the necessary corrections and adaptations

- reconciliation and preparation of the final version by the review committee

The objective of cognitive debriefing was to identify problematic questions and find solutions to make them easier to understand. To that end, 41 asthma patients were interviewed. Participants were consecutively scheduled for a single visit to the study site. At that visit, the study was explained in detail and those who agreed to participate in the study gave written informed consent. In addition, sociodemographic and specific data were collected, all of which are shown in Table 1 . The questionnaire was administered to each participant by the principal investigator. Participants were told that they need not worry about the accuracy of their responses; rather, they should report what they understood, the difficulties posed by each question or statement, and the acceptability of the instrument.

In the reconciliation step of the process, the review committee and the expert panel met in order to produce the final Brazilian Portuguese version of the ACCI, reviewing each item, discussing the findings of the cognitive debriefing process, and making the relevant changes. The final version of the ACCI for use in Brazil was thus arrived at.

Table 1. Patients participating in the cognitive debriefing process $(n=41)$, by demographic and specific characteristics. ${ }^{a}$

\begin{tabular}{lc}
\multicolumn{1}{c}{ Characteristic } & Result \\
Age, years & $39(19-86)$ \\
Female gender & $20(48.8)$ \\
Level of education & \\
$\quad$ Elementary school & $5(12.2)$ \\
$\quad$ High school & $14(34.2)$ \\
$\quad$ College & $22(53.6)$ \\
Health insurance & \\
$\quad$ Public & \\
$\quad$ Private & $21(51.2)$ \\
\hline
\end{tabular}

avalues expressed as $\mathrm{n}(\%)$, except where otherwise indicated. bValue expressed as median (minimummaximum).

\section{RESULTS}

Of the 41 asthma patients who participated in the cognitive debriefing process, 20 (48.8\%) were female. Patient age ranged from 19 years to 86 years. Most participants had a college degree (Table 1 ).

During the reconciliation, back-translation, review, and harmonization steps of the process of cultural adaptation of the ACCI, discrepancies among the individual translations (done by pulmonologists) were discussed and standardized by the review committee. In version 1 , the phrase "injeção de corticoide" was added to question 5 . The review committee made no changes to the back-translation of version 1 , version 2 being identical to version 1 . The first author of the original version approved the back-translation but raised a few questions regarding version 2: the pertinence of the phrase "um pouco incomodado(a)" in question 2 was discussed, and a decision was made to replace it with the phrase "um tanto incomodado(a)". The remaining questions raised by the original author were discussed by the review committee and did not result in changes in the text, which remained the same in version 3 and was sent to other pulmonologists for evaluation. After having discussed the comments made by those pulmonologists, the review committee prepared version 4 of the ACCI, which was sent to a teacher of Portuguese for correction. Analysis of the data collected during the cognitive debriefing process showed that there was no need to change version 4, given that the clarity index for all ACCI items was $>0.9$.

The final Brazilian Portuguese version of the ACCI incorporated changes made by the review committee because, although the ACCI was well understood (as assessed by the clarity index), a question regarding the use of corticosteroids (question 5) was often misunderstood by participants. Some of the interviewees thought that using daily inhaled corticosteroids was the same as using prednisone, whereas, in reality, daily inhaled corticosteroids and prednisone are different medications. Therefore, the review committee decided to add the sentence "(Essa pergunta não se refere a sua bombinha de uso diário)" to question 5 in order to avoid misunderstandings.

\section{DISCUSSION}

In the present study, a careful methodology was used in order to translate the ACCI and adapt it for use in Brazil. ${ }^{(15,16,18-20)}$ We decided to translate and adapt the ACCI rather than develop a new questionnaire because the ACCI, unlike other existing instruments, ${ }^{(10,11)}$ is the only instrument that was specifically developed and validated to facilitate user understanding.

The process of translation and cultural adaptation of a given questionnaire is complex but indispensable for its correct use in the target population. In addition, in the particular case of the ACCI, the translated version had to preserve the essential features of the original instrument, which was developed for ethnically diverse populations and therefore addresses social and cultural 
diversity. ${ }^{(8)}$ In Brazil, this is particularly important because the Brazilian population is heterogeneous and uses a variety of regional terms, some of which might be unknown to individuals from other regions of the country $^{(15)}$ or to immigrants from other countries. ${ }^{(16)}$ Therefore, a questionnaire should be used only in the population for which it has been developed and validated, ${ }^{(17)}$ making it possible to compare different locations and cultures in an epidemiological study. ${ }^{(15,20)}$

The Brazilian Portuguese version of the ACCI, which was arrived at in the present study, is technically and semantically equivalent to the original version. It includes terms that are commonly used in Brazil, such as the phrase "injeção de corticoide" (in question 5), which was included because corticosteroid injection is a prednisone treatment protocol that is used in the country. In addition, the statement "(Essa pergunta não se refere a sua bombinha de uso diário") was added to question 5 because users of inhaled corticosteroids commonly mistake prednisone for an inhaled corticosteroid.

Several studies have demonstrated the need for standardizing the assessment of asthma status, ${ }^{(1,8,9,21)}$ standardized assessment playing a major role in asthma control. As a result, several instruments assessing asthma status have been translated to Portuguese and adapted for use in Brazil, including the Asthma Control Questionnaire ${ }^{(11)}$ and the Asthma Control Scoring System. ${ }^{(13,21)}$ The ACCI is an important instrument because it was developed for ethnically diverse populations; it is a self-report questionnaire; and it takes only 5-7 min to complete. In addition, the response choices are sequentially color coded to quantify asthma control, thus facilitating patient understanding of asthma severity and subsequent physician management. ${ }^{(8)}$ Finally, the open-ended question allows patients to write down anything else that they consider important regarding their asthma, thus covering topics that are not covered by the remaining questions. ${ }^{(8)}$

The ACCI is an instrument that is quick and easy to implement in clinical practice because it is a self-report questionnaire designed to be completed immediately before a medical visit and because it allows physicians to focus on key aspects of disease history. In addition, the ACCI educates patients because it directs their attention to the most common signs and symptoms and allows them to recognize their severity, given that patients who do not perceive or do not recognize the severity of their symptoms are at a higher risk of exacerbations. ${ }^{(22)}$ The ACCI assesses different aspects of asthma control and takes into account the various symptoms of the disease, avoiding specific physician questions regarding each of the multiple manifestations of asthma and improving asthma care.

The ACCI addresses issues that are not addressed by other instruments and, by including questions that are more personal, such as those aimed at determining the level of asthma control and patient discomfort, allows physicians to identify the discomfort threshold in their patients and, consequently, know them better. This is important because the literature shows that a superficial assessment can result in patients with poorly controlled asthma being classified as having well-controlled asthma(23) and, consequently, receiving inappropriate treatment. This in turn can result in increased morbidity or excessive medication use and, consequently, unnecessarily increased costs. ${ }^{(24)}$

The open-ended item on the ACCI allows asthma patients to reflect on their signs and symptoms and provides physicians with an opportunity to focus on patient concerns, which are not necessarily addressed by other instruments. Therefore, the ACCI allows physicians to provide holistic care to asthma patients, as well as providing an opportunity to improve the physician-patient relationship.

Items 7 through 11 on the ACCI refer to the two weeks preceding the medical visit and follow the established international standard for questions regarding asthma symptoms. ${ }^{(5)}$ However, patient responses are more accurately classified by the ACCI than by other instruments because the ACCI provides more alternatives to be chosen from. In addition, the ACCI allows determination of the level of asthma control in accordance with the former and current Global Initiative for Asthma classifications. ${ }^{(5)}$

The ACCI was successfully translated to Portuguese and adapted for use in Brazil in accordance with international criteria. ${ }^{(16,19)}$ The Brazilian Portuguese version of the instrument is shown in Appendix 1 (available online at http://jornaldepneumologia.com. $\mathrm{br} /$ detalhe_anexo.asp?id=53). The ACCI for use in Brazil is an instrument that maintains the psychometric properties of the original questionnaire, therefore allowing comparisons of data from different countries.

\section{ACKNOWLEDGMENTS}

We would like to thank Dr. Cecilia M. Patino for allowing us to adapt the ACCI for use in Brazil and for assisting in the process of translation and cultural adaptation.

\section{REFERENCES}

1. Global Initiative for Asthma [homepage on the Internet]. Bethesda: Global Initiative for Asthma. [cited 2015 Mar 03]. Global Strategy for Asthma Management and Prevention 2006. Available from: www. ginaasthma.org

2. Instituto Brasileiro de Geografia e Estatística (IBGE). Pesquisa Nacional de Saúde 2013. Acesso e utilização dos serviços de saúde, acidentes e violências--Brasil, grandes regiões e unidades da federação. Rio de Janeiro: IBGE; 2015

3. World Health Organization [homepage on the Internet]. Geneva: World Health Organization. [cited 2014 Jan 17]. Governance [about 2 screens]. Available from: http://www.who.int/governance/en/

4. Brasil. Ministério da Saúde. Portal da Saúde [homepage on the Internet]. Brasília: Ministério da Saúde [cited 2015 Oct 18] Informações epidemiológicas de morbidade (TABNET) e mortalidade. 
Available from: http://www2.datasus.gov.br/DATASUS

5. National Institutes of Health. National Heart, Lung, and Blood Institute. National Asthma Education and Prevention Program [homepage on the Internet]. Bethesda: National Institute of Health. [cited 2015 Jun 19]. NAEPP Expert Panel Report. Guidelines for the Diagnosis and Management of Asthma--Update on Selected Topics 2015. Available from: www.nhlbi.nih.gov/guidelines/execsumm.pdf

6. Sociedade Brasileira de Pneumologia e Tisiologia. Diretrizes da Sociedade Brasileira de Pneumologia e Tisiologia para o Manejo da Asma 2012. J Bras Pneumol. 2012;38(Suppl 1):S1-S46.

7. NHLBINHO Workshop Report: global strategy for asthma management and prevention; 2002. NIH. 02-3659.

8. Patino CM, Okelo SO, Rand CS, Riekert KA, Krishnan JA, Thompson $\mathrm{K}$, et al. The Asthma Control and Communication Instrument: a clinical tool developed for ethnically diverse populations. J Allergy Clin Immunol. 2008;122(5):936-943.e6. https://doi.org/10.1016/j. jaci.2008.08.027

9. Diette GB, Patino CM, Merriman B, Paulin L, Riekert K, Okelo S, et al. Patient factors that physicians use to assign asthma treatment. Arch Intern Med. 2007;167(13):1360-6. https://doi.org/10.1001/ archinte.167.13.1360

10. Juniper EF, Buist AS, Cox FM, Ferrie PJ, King DR. Validation of a standardized version of the Asthma Quality of Life Questionnaire. Chest. 1999;115(5):1265-70. https://doi.org/10.1378/ chest.115.5.1265

11. Juniper EF, O'Byrne PM, Guyatt GH, Ferrie PJ, King DR Development and validation of a questionnaire to measure asthma control. Eur Respir J. 1999;14(4):902-7. https://doi.org/10.1034/ j.1399-3003.1999.14d29.x

12. Nathan RA, Sorkness CA, Kosinski M, Schatz M, Li JT, Marcus P, et al. Development of the asthma control test: a survey for assessing asthma control. J Allergy Clin Immunol. 2004;113(1):59-65. https:// doi.org/10.1016/j.jaci.2003.09.008

13. LeBlanc A, Robichaud P, Lacasse Y, Boulet LP. Quantification of asthma control: validation of the Asthma Control Scoring System. Allergy. 2007;62(2):120-5. https://doi.org/10.1111//.13989995.2006.01194.x

14. Tavares, MG, Pizzichini MM, Steidle LJ, Nazário NO, Rocha CC, Perraro MC, et al. The Asthma Control Scoring System: translation and cross-cultural adaptation for use in Brazil. J Bras Pneumol. 2010;36(6):683-92. https://doi.org/10.1590/S1806-

\section{4}

15. Reichenheim ME, Moraes $C L$. Operationalizing the cross-cultural adaptation of epidemiological measurement instruments [Article in Portuguese]. Rev Saude Publica. 2007;41(4):665-73. https://doi. org/10.1590/S0034-89102006005000035

16. Guillemin F, Bombardier CL, Beaton D. Cross-cultural adaptation of health-related quality of life measures: literature review and proposed guidelines. J Clin Epidemiol. 1993;46(12):1417-32. https:// doi.org/10.1016/0895-4356(93)90142-N

17. JuniperEF.Validatedquestionnaires shouldnotbe modified. Eur Respir J. 2009;34(5):1015-7. https://doi.org/10.1183/09031936.00110209

18. Wild D, Grove A, Martin M, Eremenco S, McElroy S, VerjeeLorenz, et al. Principles of Good Practice for the Translation and Cultural Adaptation Process for Patient-Reported Outcomes (PRO) Measures: report of the ISPOR Task Force for Translation and Cultural Adaptation. Value Health. 2005;8(2):94-104. https://doi. org/10.1111/j.1524-4733.2005.04054.x

19. MAPI Institute [homepage on the Internet]. Lyon: MAPI Research Institute [cited 2015 Jun 19]. Available from: http://www.mapiinstitute.com

20. Sala-Sastre N, Herdman M, Navarro L, de la Prada M, Pujol RM, Serra $\mathrm{C}$, et al. Principles and methodology for translation and cross-cultura adaptation of the Nordic Occupational Skin Questionnaire (NOSO2002) to Spanish and Catalan. Contact Dermatitis. 2009;61 (2):109-16. https://doi.org/10.1111/j.1600-0536.2009.01576.x

21. Okelo SO, Patino CM, Riekert KA, Merriman B, Bilderback $A$, Hansel $\mathrm{NN}$, et al. Patient factors used by pediatricians to assign asthma treatment. Pediatrics. 2008;122(1):e195-201. https://doi.org/10.1542/ peds.2007-2271

22. Tattersfield AE, Postma DS, Barnes PJ, Svensson K, Bauer CA O'Byrne PM, et al. Exacerbations of asthma: a descriptive study of 425 severe exacerbations. The FACET International Study Group. Am J Resp Crit Care Med. 1999;160(2):594-9. https://doi.org/10.1164/ airccm.160.2.9811100

23. Bateman ED, Bousquet $\mathrm{J}$, Braunstein $\mathrm{GL}$. Is overall asthma contro being achieved? A hypothesis generating study. Eur Respir J. 2001;17(4):589-95. https://doi.org/10.1183/09031936.01.17405890

24. Accordini S, Bugiani M, Arossa W, Gerzeli S, Marinoni A, Olivieri $\mathrm{M}$, et al. Poor control increases the economic cost of asthma. A multicentre population-based study. Int Arch Allergy Immunol. 2006:141(2):189-98. https://doi.org/10.1159/000094898 